Abstract THU0337 - Table 2

\begin{tabular}{|c|c|c|c|c|c|c|}
\hline p (significant higher parameter) & Male & Renal involvement & Lung involvement & Rheumatologic involvement & Limited vasculitis & Initial BVAS \\
\hline PR3 vs MPO & 0.71 & 0.011 (MPO) & 0.74 & 0.03 (PR3) & 0.74 & 0.38 \\
\hline GPA vs MPA & 0.09 & $<0.001$ (MPA) & 0.03 (GPA) & 0.02 (GPA) & 0.08 & 0.035 (GPA) \\
\hline MPO GPA vs PR3 GPA & 0.14 & 0.25 & 0.38 & 0.57 & 0.13 & 0.64 \\
\hline MPO GPA vs MPO MPA & $0.02(\mathrm{MPA})$ & 0.27 & 0.99 & 0.19 & $0.018(\mathrm{GPA})$ & $0.048(\mathrm{GPA})$ \\
\hline MPO MPA vs PR3 MPA & 0.52 & 1 & 0.005 (MPO) & 0.35 & 0.38 & 0.88 \\
\hline
\end{tabular}

anti-MPO GPA but relapse-free survival was lower, compared to anti-MPO MPA $(p=0.038$ and $p=0.015$, respectively), without difference in treatments. Relapsefree survival was lower in GPA, compared to MPA $(p<0.001)$. Among GPA patients, there was significantly more deaths in PR3 than MPO patients $(p=0.02)$, but without significant difference between ANCA types for all other considered criteria, including survival $(p=0.06)$.

Conclusions: The clinicopathological classification appeared as the strongest criteria for distinguish homogeneous forms and prognosis of AAV. Besides their diagnostic value, ANCA may not exhibited further great interest, especially in GPA.

Disclosure of Interest: None declared

DOI: 10.1136/annrheumdis-2017-eular.2274

\section{THU0338 CLINICAL PRESENTATION AND OUTCOMES OF EOSINOPHILIC GRANULOMATOSIS WITH POLYANGIITIS: ANCA-NEGATIVE VERSUS ANCA-POSITIVE}

E. Zagvozdkina, P. Novikov, S. Moiseev. Sechenov First Moscow State Medical University, Moscow, Russia, Moscow, Russian Federation

Objectives: The aim of the prospective study was to compare clinical presentation at diagnosis and long-term outcomes of eosinophilic granulomatosis with polyangiitis (Churg-Strauss) (EGPA) patients according to antineutrophil cytoplasmic antibody (ANCA) status.

Methods: EGPA was classified according to the Revised CHCC Nomenclature and ACR1990 criteria. Activity of vasculitis was evaluated using BVAS. A minor relapse was defined as an increase in at least one new or worse minor item and no major BVAS items. A major relapse was an increase in at least one major BVAS item.

Results: We followed 93 patients with EGPA for a mean \pm SD of $6.3 \pm 6.5$ years. Their mean \pm SD age was $46.6 \pm 13.8$ years, and $96.8 \%$ patients had a history of asthma. The most common EGPA manifestations at diagnosis included ENT manifestations (88.2\%), fever (78.5\%), peripheral neuropathy $(73.1 \%)$, lung involvement $(59.1 \%)$ and skin lesions (49.5\%). Thirty seven of 93 patients $(39.8 \%)$ were ANCA-positive. These patients had significantly more frequent myalgia and mononeuritis multiplex, than the ANCA-negative patients. The difference in occurrence of kidney disease between the two groups did not reach statistical significance. However, all three patients with rapidly progressive kidney failure were ANCA-positive. BVAS at diagnosis and VDI at the end of the follow-up were significantly higher for ANCA-positive patients. The follow up duration was 587.7 patient-years. The incidence of all vasculitis relapses was 14 per 100 patient-years, including major and minor vasculitis relapses (5.3 and 8.7 per 100 patient-years, respectively). The 3- and 5-year relapse-free survival rate was $65.4 \%(95 \% \mathrm{Cl} 54.6-76.2)$ and $43.1 \%(95 \% \mathrm{Cl} 30.4-55.7)$, respectively. The frequency of vasculitis relapses was 21.0 per 100 patient-years in the ANCA-positive group versus 19.6 per 100 patient-years in the ANCA-negative group $(\mathrm{P}=0.4)$.

Table 1. Main clinical characteristics at diagnosis of the 71 patients with EGPA, according to ANCA status

\begin{tabular}{lccc}
\hline Manifestations & ANCA $+(\mathrm{n}=37)$ & ANCA- $(\mathrm{n}=34)$ & $\mathrm{P}$ \\
\hline Fever & $30(81.1)$ & $25(73.5)$ & 0.57 \\
Myalgia & $22(59.5)$ & $10(29.4)$ & $\mathbf{0 . 0 2}$ \\
ENT & $33(89.2)$ & $30(88.2)$ & 1.00 \\
Lung & $24(64.9)$ & $19(55.9)$ & 0.47 \\
Cutaneous & $24(64.9)$ & $15(44.1)$ & 0.10 \\
Peripheral neuropathy & $30(81.1)$ & $21(61.8)$ & 0.11 \\
Mononeuritis multiplex & $17(45.9)$ & $6(17.6)$ & 0.01 \\
Cardiovascular & $13(35.1)$ & $10(29.4)$ & 0.62 \\
Gastrointestinal & $4(10.8)$ & $4(11.8)$ & 1.00 \\
Renal & $11(29.7)$ & $5(14.7)$ & 0.16 \\
BVAS at diagnosis, mean \pm SD & $16.86 \pm 6.44$ & $12.62 \pm 5.52$ & $<\mathbf{0 . 0 1}$ \\
VDI at the end of the follow-up & $2.43 \pm 1.57$ & $1.76 \pm 1.16$ & $<\mathbf{0 . 0 1}$ \\
\hline
\end{tabular}

Conclusions: The characteristics of EGPA patients differ according to their ANCA status, although long-term outcomes were similar in both groups. EGPA is characterized by relapsing course of disease. The minor vasculitis relapses predominated in the structure of relapses.

Disclosure of Interest: None declared

DOI: 10.1136/annrheumdis-2017-eular.5259

\section{THU0339 FECAL CALPROTECTIN LEVELS AS AN INDICATOR OF ACTIVE DISEASE IN BEHÇET'S SYNDROME PATIENTS WITH GASTROINTESTINAL INVOLVEMENT: A CONTROLLED STUDY}

S.N. Esatoglu ${ }^{1}$, I. Hatemi ${ }^{2}$, Y. Ozguler ${ }^{1}$, G. Hatemi ${ }^{1}$, H. Uzun ${ }^{3}$, A.F. Celik ${ }^{2}$ H. Yazici ${ }^{1} .{ }^{1}$ Istanbul University, Cerrahpasa Medical School, Department of Internal Medicine, Division of Rheumatology; ${ }^{2}$ Istanbul University, Cerrahpasa Medical School, Department of Internal Medicine, Division of Gastroenterology; ${ }^{3}$ Istanbul University, Cerrahpasa Medical School, Department of Biochemistry, Istanbul, Turkey

Background: Elevated fecal calprotectin (FC) levels indicate activity in Crohn's disease (CD) and ulcerative colitis and are used as non-invasive biomarkers in these diseases. Gastrointestinal involvement of Behçet's syndrome (GIBS) shows clinical and endoscopic similarities to CD. A previous study suggested that FC may help to diagnose GIBS patients (1), but we are not aware of any studies addressing its role in identifying disease activity in such patients.

Objectives: To determine whether FC helps to distinguish active GIBS patients from those in remission.

Methods: We collected fecal and serum specimens before colonoscopy from 39 GIBS patients who agreed to participate (Table). Twenty-six patients were asymptomatic whereas 13 had abdominal pain and/or diarrhea. We also filled disease activity index for intestinal Behçet's disease (DAIBD) and Crohn's disease activity index (CDAI) in each patient. Active gastrointestinal (GI) involvement was defined as having ulcers on colonoscopy. We included 22 active and 25 inactive CD patients as controls. We used $150 \mu \mathrm{g} / \mathrm{g}$ as the cut-off for a positive FC level. None of the patients were receiving NSAIDs that could increase FC levels.

Results: Among the 39 GIBS patients, 14 had active ulcers on colonoscopy (8/13 symptomatic and 6/26 of asymptomatic). FC level was $>150 \mu \mathrm{g} / \mathrm{g}$ in 12/14 active GIBS patients and in 6/25 patients in GI remission (OR: $19,95 \% \mathrm{Cl}: 3$ to 110). The median FC and CRP levels were higher among active GIBS patients whereas serum calprotectin levels were not different (Table). Among CD patients, $16 / 25$ active patients and $3 / 22$ patients in remission had a FC level $>150 \mu \mathrm{g} / \mathrm{g}$ (OR: $11,95 \% \mathrm{Cl}: 11$ to 49 ). There was a high correlation between FC and CDAl scores $(r=0.64, p<0.001)$ and a very high correlation between FC and DAIBD scores $(r=0.71, p<0.001)$, while $F C$ was not correlated with serum calprotectin and CRP levels. Among the 6 GIBS patients who had high FC levels despite being in remission for Gl involvement, 2 had active mucocutaneous lesions, 1 had concomitant macrophage activation syndrome (MAS), 1 had polycythemia vera with trisomy 8 and 2 were started high dose corticosteroids. Repeat FC levels could be obtained in 3 of these patients, after the resolution of MAS and mucocutaneous lesions, and were $<150$ in all 3 .

Table 1. Demographic, clinical and laboratory features of active GIBS patients and in GIBS patients who are in remission

\begin{tabular}{lccc}
\hline & $\begin{array}{c}\text { Active GIBS } \\
(\mathrm{n}=14)\end{array}$ & $\begin{array}{c}\text { GIBS in remission } \\
(\mathrm{n}=25)\end{array}$ & $P$ value \\
\hline Gender, M/F & $5 / 9$ & $10 / 15$ & 0.15 \\
Mean \pm SD age, years & $47.5 \pm 7.2$ & $40 \pm 11$ & 0.03 \\
Median (IQR) FC, $\mu \mathrm{g} / \mathrm{g}$ & $301(176-957)$ & $30(30-134)$ & $<0.001$ \\
$\mathrm{FC} \geq 150 \mu \mathrm{g} / \mathrm{g}, \mathrm{n}(\%)$ & $12(86)$ & $6(24)$ & $<0.001$ \\
Median (IQR) serum calprotectin, $\mathrm{ng} / \mathrm{mL}$ & $98(39-128)$ & $69(54-101)$ & 0.5 \\
Median (IQR) serum CRP, $\mathrm{mg} / \mathrm{dL}$ & $2(1-5)$ & $3(2-15)$ & 0.07 \\
Median (IQR) CDAI scores & $52(4-189)$ & $30(0-92)$ & 0.2 \\
Median (IQR) DAIBD scores & $40(4-81)$ & $20(0-37)$ & 0.1 \\
\hline
\end{tabular}

Conclusions: FC seems to be a useful non-invasive tool for identifying active GI involvement in GIBS patients. Whether the presence of other BS manifestations can cause false positive results in GIBS patients in remission remains to be studied. On the other hand, serum calprotectin levels do not seem to be useful in identifying active disease in GIBS patients.

References:

[1] Kim DH et al. J Gastroenterol Hepatol. 2016.

Disclosure of Interest: None declared

DOI: 10.1136/annrheumdis-2017-eular.6124 